\title{
Motor Knowledge Modulates Attentional Processing during Action Judgment
}

\author{
By Christel Bidet-Ildei* \\ Cedric Bouquet ${ }^{\dagger}$
}

\begin{abstract}
Several studies have revealed the role of motor experience when humans have to judge human actions. However, the possible influence of motor knowledge on attention processes has been neglected. This study used a flanker paradigm (Eriksen and Eriksen 1974) to analyze the attention responses of male and female participants during the judgment of running movements. Three running actions appeared simultaneously on a computer screen, with the target in the center and the flankers in the periphery. The target and flankers could be compatible or incompatible concerning both the direction of the movement and the sex of the runner. Moreover, flankers were presented upright or upside-down. The results indicate that the distracting effect of the flankers was different for male and female participants. Whereas direction-incompatible flankers systematically disturbed the female participants' performance, regardless of the sex and the orientation of the flanker, male participants were only distracted when the flankers were upright males. This finding offers new directions to explain sex differences in the judgment of human action.
\end{abstract}

Keywords: action observation, attention, motor repertoire, sex differences

\section{Introduction}

The high sensitivity of the visual system to human actions is well known and is implicated in many survival behaviors, such as interpreting emotions or predicting the future actions of others (for recent reviews see, Rizzolatti and Fabbri-Destro 2008, Blake and Shiffrar 2007). One explanation for this sensitivity is based on a motor-perceptual relationship, such that the observation of action involves both the motor and the perceptual systems (Calvo-Merino et al. 2006, Schütz-Bosbach and Prinz 2007, Jeannerod 2001). Many psychophysical, neuroimaging and neuropsychological studies argue in favor of this theory by showing common characteristics in the production of motor action and the qualitative judgment of similar action that is passively

${ }^{*}$ CeRCA, University of Poitiers, France.

${ }^{\dagger}$ CeRCA, University of Poitiers, France. 
observed. Positron emission tomography and functional magnetic resonance imaging studies have revealed the activation of overlapping brain areas when comparing actual movement production with the observation of similar movements (Decety et al. 1997, Hari et al. 1998, Nishitani and Hari 2000, Rizzolatti et al. 1996a, Rizzolatti et al. 1996b). For instance, in the context of a manual reaching task, the parietal, premotor and motor areas were found to be activated during both the production and the observation of reaching movements (Filimon et al. 2007). Reported neuropsychological cases have also shown specific correspondences between deficit in the production and observation of action (Chary et al. 2004, Sirigu et al. 1995).

Interestingly, motor area activation is greater when the observed motor sequence belongs to the observer's motor repertoire (Calvo-Merino et al. (2005, 2006). Moreover, behavioral level, recognition, discrimination and anticipation are improved when the observed action belongs to the observer's motor repertoire (Calvo-Merino et al. 2009, Casile and Giese 2006, Beardsworth and Buckner 1981, Loula et al. 2005, Louis-Dam et al. 2000, Martel et al. 2011). For example, the capacity to discriminate non-biological vs. biological locomotion was found to be significantly affected by non-visual motor practice (Casile and Giese 2006). In the same manner, predictive performance increases when participants have to judge their own movements, as opposed to movements of other individuals (e.g., Martel et al. 2011). This effect of motor repertoire was also demonstrated by varying characteristics of human movements (Calvo-Merino et al. 2009). Especially, authors have compared the discrimination of experts (dancers) and control observers during the judgments of dancing movements presented in upright or inverted orientation. Results indicated better performance for experts when judging upright stimuli whereas there was no difference with non-experts when judging the same stimuli presented upside-down (Calvo-Merino et al. 2009). This confirms previous investigations, which demonstrate that stimulus orientation affects the capacity to judge human movements (Pavlova and Sokolov 2000, Shipley 2003), and suggests that the observer's motor repertoire does not intervene during the judgments of inverted human movements. Finally, a recent experiment (Bidet-Ildei et al. 2010) also revealed that observing or producing a running activity during a very short period can both improve the subsequent visual processing of similar point-light human actions. However, the positive effect of prior observation was dependent on the congruency between the sex of the observers and the sex of the observed actor, suggesting that the motor repertoire differed in male and females. Considered together, these data strongly support a common representation between perception and action (i.e., Hommel et al. 2001). Moreover, these data suggest that motor experience influences the perception of human action.

Thus, several experiments have demonstrated the influence of motor knowledge on the recognition (Loula et al. 2005), discrimination (CalvoMerino et al. 2009, Casile and Giese 2006), and anticipation of observed actions (Martel et al. 2011, Chary et al. 2004, Louis-Dam et al. 2000). However, the possible influence of motor knowledge on attention processes 
during action perception has been neglected. In the present study, we addressed this question by adapting the flanker paradigm (Eriksen and Eriksen 1974) to the observation of human actions.

Classically, in the flanker task, participants are asked to process a central target stimulus while ignoring the distracting information flanking the critical target. A flanker-compatibility effect (FCE) is usually observed, with increased reaction times (RT) and error rate when the distractor and the target stimulus are associated with incompatible responses. The flanker task is intended to assess the subject's ability to resist or resolve interference from distracting information. This resistance to distractor interference, indexed by the FCE, has been associated with focused attention on target stimuli and the active suppression of irrelevant information (Eriksen and Eriksen 1974, Kok 1999).

In the present study, we had participants judge the direction of running actions, presented as a central target, against two other running actions, presented as flankers. The target and flankers could be compatible or incompatible with respect to the direction of running movement. As seen above, various manipulations allow the correspondence between the observer's motor competences and the observed action, to vary- hence modifying the possibility to use motor knowledge and influencing the judgment of human action (Beardsworth and Buckner 1981, Bidet-Ildei et al. 2010, Bidet-Ildei et al. 2011, Calvo-Merino et al. 2009, Calvo-Merino et al. 2006, Loula et al. 2005, Martel et al. 2011). In order to address the influence of stored motor representations on visual attention processes we first manipulated the orientation of the flankers, with distracting running movements being presented upright or upside-down. To further evaluate this influence of motor repertoire, we also manipulated the congruence between the participants' gender and the flankers' gender. We included male and female participants, and either a male or a female actor performed the running movements presented as flankers. Interestingly, this manipulation also permitted us to test for potential sex differences. It has been demonstrated that men and women differ in action perception, with women outperforming men in tasks involving judgment or understanding of action (e.g. Cheng et al. 2006). Therefore, we will also test for possible differences between men and women in visual attention to human movements.

We thus had female and male participants judge the direction of a running action, presented as a central target, flanked by two other running actions. We expected a classical FCE with increased RT when directions of flankers and the target were incompatible. If stored motor representations are involved in these attention effects, both the flanker's gender and orientation should modulate the FCE effects. We expected reduced FCE for inverted flankers. In the same way, we expected the FCE to be stronger when the flankers' gender matches the participant's gender.

Finally, it is known that changing the orientation of biological movement produces a critical decrease in human recognition performance (e.g., Pavlova and Sokolov 2000). To control this effect and assess the potential role of explicit recognition in the main task, we added a complementary task in which 
participants had to recognize explicitly the sex of the actor performing the actions presented as flankers.

\section{Method}

\section{Participants}

Twenty-four healthy volunteers (12 females, 12 males) between 19 and 26 years of age $(M=20.95$ years, $S D=1.92$ years $)$ participated in the experiment for course credits. None of the participants reported any sensory or motor deficits, and all participants had normal or corrected-to-normal vision. In accordance with the Helsinki declaration, all participants provided their informed consent to participate.

Two participants were excluded due to error rates exceeding $30 \%$, leaving 22 participants (11 females, 11 males), between 19 and 26 years of age $(\mathrm{M}=21.04$ years, $\mathrm{SD}=1.99$ years $)$.

\section{Material and Stimuli}

Participants were seated in a dimly lit room in front of a CRT computer screen (19", spatial resolution: $1024 * 768$ pixels, sampling rate: $85 \mathrm{~Hz}$ ) at a viewing distance of $50 \mathrm{~cm}$. The visual angle of the screen subtended at eye-level was $32^{\circ}$ vertical $(\mathrm{V}) * 41.4^{\circ}$ horizontal $(\mathrm{H})$. Body movements were limited by the contact of the chest with the table supporting the computer screen.

The stimulus was composed of three video sequences, each showing an excerpt of male or female running movements (see figure 1). Each video (spatial resolution: $320 * 240$ pixels, sampling rate: 25 frames/s) occupied a window of $9.2^{\circ}(\mathrm{V}) * 13.06^{\circ}(\mathrm{H})$ of the visual angle and showed a male or a female in profile $\left(4.4^{\circ}(\mathrm{V}) * 2.3^{\circ}(\mathrm{H})\right)$ who produced one step of a running movement in the left or right direction. The displacement occupied $3.02^{\circ}(\mathrm{H})$ of the visual angle and had duration of $600 \mathrm{~ms}$. Except the actor's sex, all low visual characteristics were controlled (see Figure 1). Male and female runners were dressed similarly. Moreover, each running movement was cut and pasted in a neutral white visual environment. Finally, the woman's hair was pulled up to obtain, as much as possible, low visual cues with respect to the male runner. In the upright conditions, flankers were presented with a normal orientation, namely the orientation observed when humans run. In the inverted conditions, the same stimuli were presented. The participants were asked to concentrate exclusively on the video sequence presented in the center of the screen, considered as the target, while ignoring the flankers. At the top of the stimulus, the target and the flankers were separated by a visual angle of $13.3^{\circ}(\mathrm{H})$. The flankers were always identical and were related to the target by sex $*$ direction compatibility (or incompatibility), resulting in 2 (target's sex: male, female) * 2 (target's direction: left, right) * 2 (flankers' sex: male, female) * 2 (flankers' direction: left, right) * 2 (flanker's position: upright, inverted) possible combinations. 
The presentation of stimuli and the registration of manual responses were controlled by E-prime software (version 2.0, http://www.pstnet.com/). An Eprime SRbox response device was positioned on a table close to the body of the participants so they could easily give their response by pressing one of two keys.

Figure 1. Static Presentation of One Condition. The Target was the Woman and the Flankers were the Men. In this Condition, we had s Compatibility of Direction but no Perceptual Similarity. For Male Participants (but not Female Participants), we also had a Motor Flanker Similarity

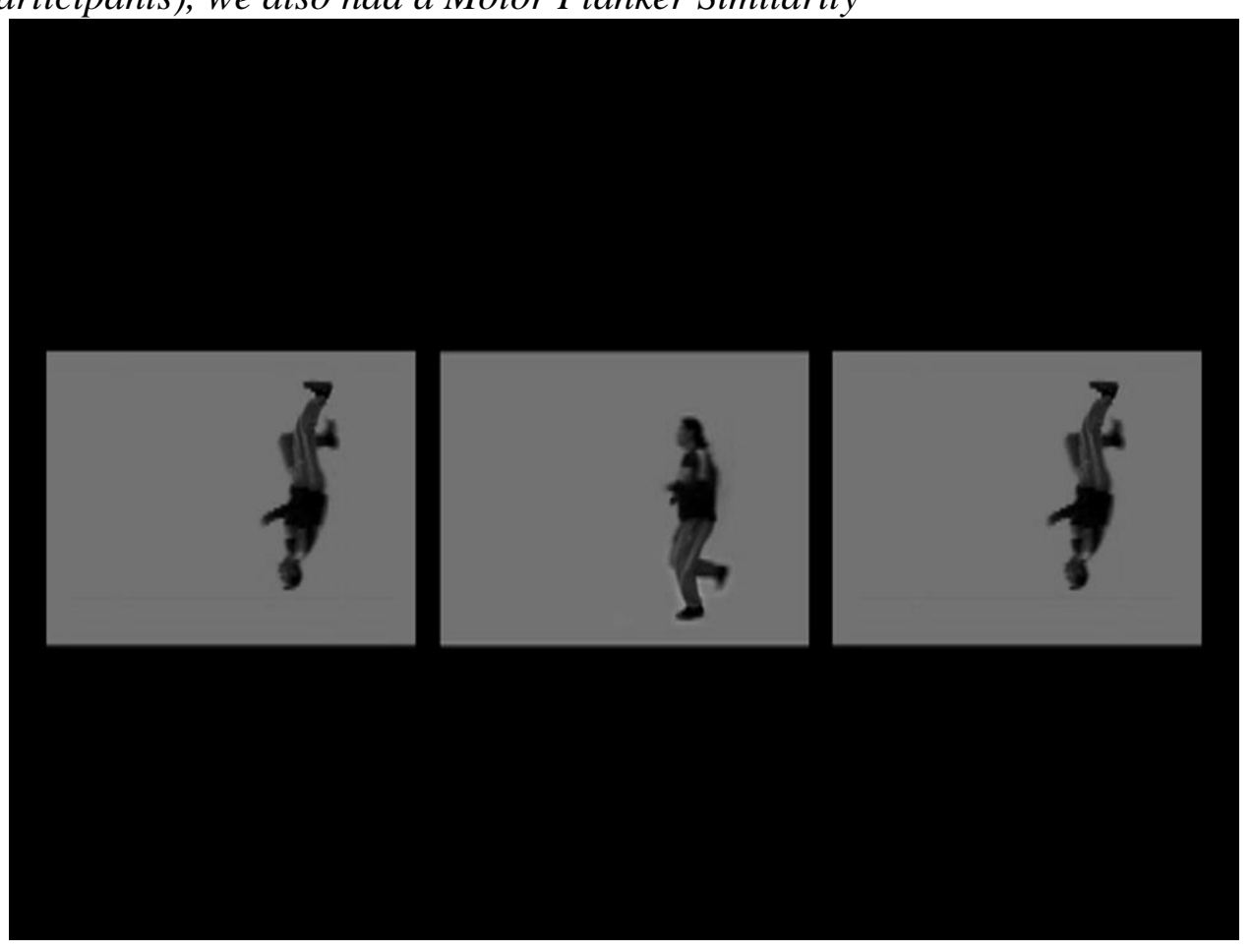

\section{Procedure}

Each trial began with the presentation of a fixation cross at the center of the screen for $250 \mathrm{~ms}$, followed by the stimulus. The task consisted of indicating the direction (left or right) of the target displacement as quickly and accurately as possible, by pressing with the right or left hand index finger a response keysituated on the right (for the right direction) or left side (for the left direction) of the button box. The experiment was organized in eight sessions of 96 trials each ( 3 trials $* 32$ combinations), resulting in a total duration of approximately 25 minutes per experimental session.

This main part of the experiment was followed by a final block of 32 trials (one in each condition) in which the participants performed the same task, but after each trial, they were asked to decide whether the flankers were males or females. This procedure was used to assess explicit recognition of the flankers. 


\section{Data Analysis}

Accuracy and reaction time (RT) were evaluated for each subject and each trial and were averaged for each participant. Given the distribution of RTs, the analyses were based on the log transformation of the RTs for correct responses in a cell. Trials following an error were excluded from this analysis. Moreover, for each participant, we removed the RTs farther than three standard deviations away from the mean (4.5\% in average). The accuracy and reaction time were statistically evaluated using a five-way analysis of variance (ANOVA) with the participants' sex (male/female) as the between-subject factor and the targetflanker direction compatibility (compatible/incompatible), target's sex (male/ female), flankers' sex (male/female) and flankers' orientation (upright/ inverted) as within-subject factors. If the sphericity assumption was violated (i.e., presented an epsilon less than 1), Huyn-Feldt adjustments of the p-values were performed. Local comparisons were performed using post-hoc Duncan's comparisons. Effect sizes were computed using eta-square estimates.

For the second part of the experiment, assessing recognition, we computed the percentage of correct recognition for upright male, upright female, inverted male and inverted female flankers for male and female participants ( 8 trials per category). We conducted a statistical analysis with an ANOVA with the flanker's orientation and sex as repeated factors and the participant's gender as a between subject factor. In this analysis, we kept only correct responses for RT between 100 and $600 \mathrm{~ms}$, to limit a possible change of strategy due to the recognition task.

\section{Results}

With respect to accuracy (see Figure 2), we observed a classical FCE $\left(F(1,20)=19.78 ; p<0.01 ; \eta^{2}=0.95\right)$, which was characterized by a decrease of correct responses when target and flankers had incompatible directions $(\mathrm{M}=$ $80.9 \%, \mathrm{SD}=8.2 \%)$ in comparison with compatible directions $(\mathrm{M}=84.1 \%, \mathrm{SD}$ $=6.4 \%$ ). No other significant simple effect or interaction indicating sex differences appeared in the analysis. 
Figure 2. Mean and Standard Error of Percentage of Correct Responses for Upright $(A \& B)$ and Inverted Conditions $(C \& D)$ in Congruent and Incongruent Direction Conditions and for Female and Male Participants
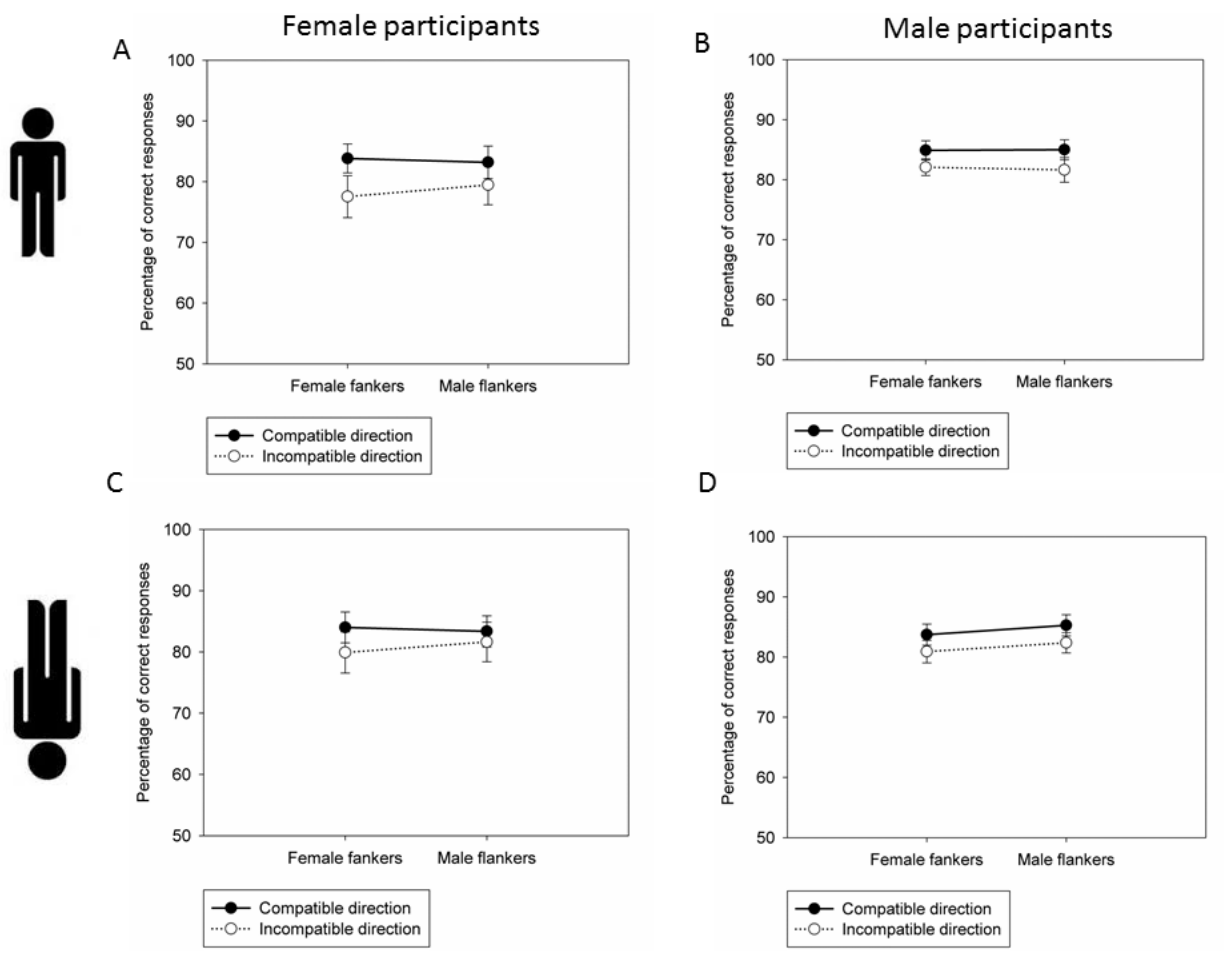

With respect to RT (see Figure 3), we observed the same presence of FCE, which was characterized by an increase of RT $\left(F(1,20)=28.9 ; p<0.0001 ; \eta^{2}\right.$ $=0.97$ ) with incompatible directions between the target and flankers $(\mathrm{M}=$ $331.4 .3 \mathrm{~ms}, \mathrm{SD}=21.8 \mathrm{~ms})$ in comparison with compatible directions $(\mathrm{M}=$ $325.1 \mathrm{~ms}, \mathrm{SD}=19.4 \mathrm{~ms})$. Moreover, we obtained a significant effect for the target's sex $\left(\mathrm{F}(1,20)=12.27 ; \mathrm{p}<0.01 ; \eta^{2}=0.92\right)$. In both female and male participants, RT was higher for female targets $(\mathrm{M}=329.9 \mathrm{~ms}, \mathrm{SD}=20.6 \mathrm{~ms})$ than male targets $(\mathrm{M}=326.5 \mathrm{~ms}, \mathrm{SD}=20.4 \mathrm{~ms})$. Interestingly, we observed a significant interaction between the participant's sex, target-flanker direction compatibility, the flankers' sex and the flankers' orientation $(\mathrm{F}(1,20)=6.73$; $\mathrm{p}<0.05 ; \eta^{2}=0.87$ ). To clarify this interaction (see figure 2 and 3 ), we performed additional ANOVAs in women and men, separately.

In women, we observed no interaction implicating flankers' sex or orientation, indicating that the FCE was similar regardless of the sex or the orientation of the flankers (all Fs <1).

Contrarily, in men, there was a significant interaction between targetflanker direction compatibility and the flankers' orientation $(\mathrm{F}(1,10)=5.04$; $\left.\mathrm{p}<0.05 ; \eta^{2}=0.83\right)$. The Post-Hoc analysis indicates that male participants were influenced by direction compatibility in upright conditions $(\mathrm{p}<0.01)$ but not in inverted conditions $(\mathrm{p}=0.67)$. This interaction was also modulated by the flankers' sex $\left(F(1,10)=11.33 ; p<0.01 ; \eta^{2}=0.91\right)$. 
Figure 3. Mean and Standard Error of Reaction Time in Upright (A and B) and inverted ( $C$ and $D)$ Conditions for Congruent and Incongruent Direction Conditions and for Female and Male Participants. Asterisk Indicates Significant Difference at $p<0.05$
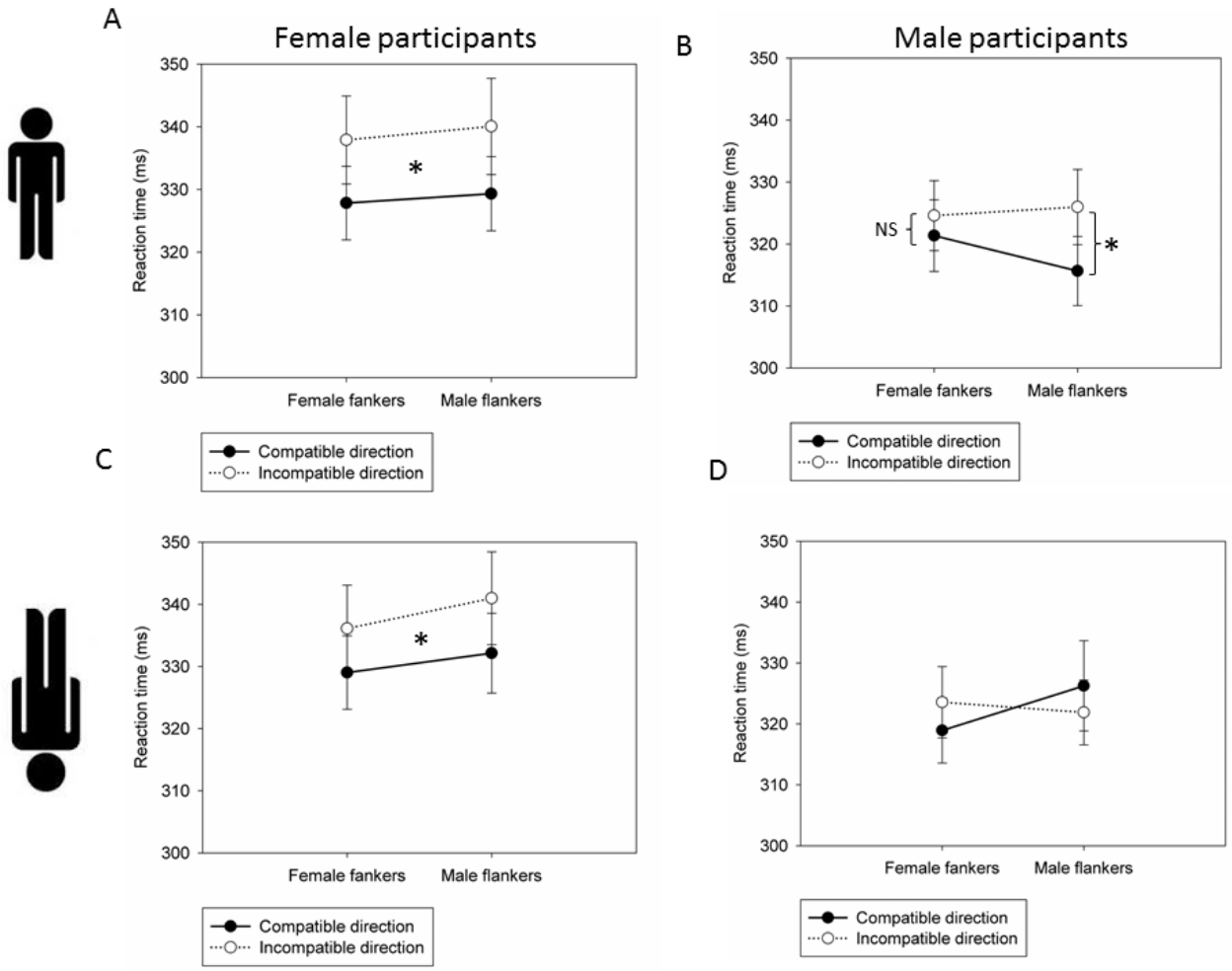

A post-hoc analysis showed that FCE was present when the flankers were upright males $(\mathrm{p}<0.01)$ but not when the flankers were upright females $(\mathrm{p}=$ $0.23)$. No FCE was observed with inverted flankers $(p=0.11$ and $p=0.13$, for inverted females and inverted males respectively).

Concerning the second part of the experiment (assessing identification of flankers' sex $)$, the analysis revealed an effect of orientation $(F(1,20)=36.32$; $\left.\mathrm{p}<0.01 ; \eta^{2}=0.97\right)$. Percentage of correct recognition was higher for upright flankers $(\mathrm{M}=84.6 \%, \mathrm{SD}=12.6 \%)$ than inverted flankers $(\mathrm{M}=66.5 \%, \mathrm{SD}=$ $14.6 \%)$. There were no other significant effects.

\section{Discussion}

In the current work, we found classical flankers' interference when the target and the flankers were associated with incompatible responses. These findings extend the results obtained by Thornton and Vuong (2004) by showing that the FCE can appear not only in the judgments of point-light biological motion but also in the observation of video sequences representing real human actions.

Most importantly, the present study suggests that the participants' sex and motor competencies modulate the FCE. In the upright condition, the RT data 
indicate that both female and male flankers distracted women, whereas only male flankers distracted male participants. Moreover, in inverted conditions, the FCE persisted in women for both male and female flankers, whereas it disappeared in male participants. The significant increases/decreases of RT were not associated with any decrease/increase of correct responses, excluding a speed-accuracy trade-off.

These findings contrast with those obtained by Thorton and Vuong (2004) during observations of point-light biological motion by showing that human movements are not systematically treated incidentally. Our results are more in accordance with the intervention of attention during the perception of biological motions (see Thompson and Parasuraman, 2011 for a review). In the present study, the influence of human movements presented as distractors appeared to depend on the sex of the observer. Notably, the systematic processing of the distractor, indexed by the FCE, was observed only in females. In contrast, in males, the FCE was present only when the sex of the participants matched the sex of the distractors. This finding extends the recent results obtained by Stoet (2010) by showing sex differences in the processing of irrelevant information in the context of human movement observation.

Several experiments have shown that the motor repertoire can influence a subject's judgments of human action (Bidet-Ildei et al. 2010, Casile and Giese 2006, Calvo-Merino et al. 2005). The present study reveals that men were attracted by irrelevant information only when the distracters were upright males. Actually, one important feature of the upright male flankers is that they correspond to the characteristics of male motor running experience. Men were thus disturbed only by flanker movements that matched their motor repertoire. This effect suggests that motor experience could affect attention processing of human action, at least in men.

However, the use of motor repertoire cannot account for the findings obtained in women. This observation is surprising and strongly suggests the existence of sex differences in attention processing during the visual judgments of human action. Actually, the present result confirms a preliminary investigation conducted in our lab, which showed that men made more errors in a flanker task when flankers were male runners whereas women were disturbed in the same manner by female and male flankers (Bidet-Ildei and Bouquet 2011). It seems that biological movements can automatically capture women's attention, even when the information is irrelevant for the task and even when the perceived action does not match the women's motor experience. We can interpret this finding as an automatic attraction to biological movements and/or specific difficulties to inhibit treatment of irrelevant biological movements. Contrarily, in men, the inhibition of irrelevant stimuli seems more efficient, except in conditions where the flankers correspond to their motor repertoire.

It is also possible that differences in recognition of flankers also contributed to the present pattern of results. Women may have been distracted by incompatible male and female flankers partly because they recognized both types of stimulus. In contrast, in the male participants, the restriction of the 
FCE to the upright male flankers may be partly explained by a better recognition of these distractors. The results obtained in the second part of the experiment do not agree with this assumption. Actually, there was no effect of the participants' sex or flankers' sex in recognition performance. Of course, caution should be taken here because the assessment of recognition is not completely identical to the task where the FCE was evaluated. Nevertheless, these findings clearly challenge the idea that explicit recognition can account for sex differences in the FCE.

Interestingly, sex differences in attention processes could clarify the sex differences usually obtained during the observation and judgments of human actions (Cheng et al. 2009, Cheng et al. 2007, Cheng et al. 2008, Cheng et al. 2006, Pavlova et al. 2010a). Early experiments on facial recognition showed that women outperformed men in recognizing facial expressions and facial intentions (Farris et al. 2008, Montagne et al. 2005). Magnetoencephalography (MEG) investigations have also revealed differences between males and females in predicting social interactions (Pavlova et al. 2010). Although similar behavioral performance was observed, gamma oscillatory activation in the left prefrontal cortex appeared earlier in females than in males, indicating that females are able to make social decisions faster than males. Some potential explanations, such as neuroanatomical (e.g., Cheng et al. 2009), neurobiological differences (e.g., Cahill 2006) or even discrepancies in stereotype susceptibility (Pavlova et al. 2010b) have been evoked as sources of sex differences in the prediction or understanding of human actions. For example, by using a voxel-based morphometric approach, Cheng and his colleagues (2009) demonstrated differences in the pars opercularis and the inferior parietal lobule between women and men and found that these differences correlated with empathetic performance. The present results may indicate that the better performance observed in women concerning the judgments of human movements could partially emerge from differences in attention processes. If women are more sensitive (in terms of attention) to human movements, they may be more competent in tasks requiring the processing of human actions. This hypothesis should be specifically tested in future experiments.

As a whole, this study strongly suggests that the attention processes involved in the observation of human movements 1) differ between males and females and 2) depend on the motor repertoire of the observers, particularly in men. Future studies should determine the neurophysiological support for this sex discrepancy in the attention processing of human actions.

\section{Acknowledgments}

We thank all participants for their participation and Yves Almecija for his help in the construction of the stimuli. This work was supported by the National Center of Scientific Research (CNRS, UMR 7295). 


\section{References}

Beardsworth T, Buckner T (1981) The ability to recognize oneself from a video recording of one's movements without seeing one's body. Bulletin of the Psychonomic Society 18(1): 19-22.

Bidet-Ildei C, Bouquet CA (2011) Flanker's paradigm reveals attention gender differences in action's judgments. In PERCEPTION, ed. Perception (ECVP), 2011 Toulouse (France): 15.

Bidet-Ildei C, Chauvin A, Coello Y (2010) Observing or producing a motor action improves later perception of biological motion: Evidence for a gender effect. Acta Psychologica (Amst) 134(2): 215-224.

Bidet-Ildei C, Kitromilides-Salerio E, Orliaguet JP, Badets A (2011) Perceptual Judgements of Handwriting and Pointing Movements: Influence of Kinematics Rules. In AM Columbus (Ed) Advances in Psychology Research. New York: Nova Publisher.

Blake R, Shiffrar M (2007) Perception of human motion. Annual Review of Psychology 58: 47-73.

Cahill L (2006) Why sex matters for neuroscience. Nature Reviews Neuroscience 7(6): 477-84.

Calvo-Merino B, Ehrenberg S, Leung D, Haggard P (2009) Experts see it all: configural effects in action observation. Psychological Research 74(4): 400-6.

Calvo-Merino B, Glaser DE, Grezes J, Passingham RE, Haggard P (2005) Action observation and acquired motor skills: an FMRI study with expert dancers. Cerebral Cortex 15(8): 1243-9.

Calvo-Merino B, Grezes J, Glaser DE, Passingham RE, Haggard P (2006) Seeing or doing?. Influence of visual and motor familiarity in action observation. Current Biology 16(19): 1905-10.

Casile A, Giese MA (2006) Nonvisual motor training influences biological motion perception. Current Biology 16(1): 69-74.

Chary C, Meary D, Orliaguet JP, David D, Moreaud O, Kandel S (2004) Influence of motor disorders on the visual perception of human movements in a case of peripheral dysgraphia. Neurocase 10(3): 223-32.

Cheng Y, Chou KH, Decety J, Chen IY, Hung D, Tzeng OJ, Lin CP (2009) Sex differences in the neuroanatomy of human mirror-neuron system: a voxel-based morphometric investigation. Neuroscience 158(2): 713-20.

Cheng Y, Decety J, Lin CP, Hsieh JC, Hung D, Tzeng OJ (2007) Sex differences in spinal excitability during observation of bipedal locomotion. Neuroreport 18(9): 887-90.

Cheng Y, Lee PL, Yang CY, Lin CP, Hung D, Decety J (2008) Gender differences in the mu rhythm of the human mirror-neuron system. PLOS ONE 3(5): 2113.

Cheng Y, Tzeng OJ, Decety J, Imada T, Hsieh JC (2006) Gender differences in the human mirror system: a magnetoencephalography study. Neurorepor 17(11): $1115-1119$.

Decety J, Grezes J, Costes N, Perani D, Jeannerod M, Procyk E, Grassi F, Fazio F (1997) Brain activity during observation of actions. Influence of action content and subject's strategy. Brain 120 (Pt 10): 1763-77.

Eriksen BA, Eriksen CW (1974) Effects of noise letters upon the identification of a target letter in a non-search task. Perception \& Phychophysics 16: 143-149.

Farris C, Treat TA, Viken RJ, Mcfall RM (2008) Perceptual mechanisms that characterize gender differences in decoding women's sexual intent. Psychological Science 19(4): 348-54. 
Filimon F, Nelson JD, Hagler DJ, Sereno MI (2007) Human cortical representations for reaching: mirror neurons for execution, observation, and imagery. Neuroimage 37(4): 1315-28.

Hari R, Forss N, Avikainen S, Kirveskari E, Salenius S, Rizzolatti G (1998) Activation of human primary motor cortex during action observation: a neuromagnetic study. Proceedings of the National Academy of Sciences USA 95(25): 15061-5.

Hommel B, Musseler J, Aschersleben G, Prinz W (2001) The Theory of Event Coding (TEC): a framework for perception and action planning. Behavioral Brain Sciences 24(5): 849-78, discussion 878-937.

Jeannerod M (2001) Neural simulation of action: a unifying mechanism for motor cognition. Neuroimage 14(1 Pt 2): S103-9.

Kok A (1999) Varieties of inhibition: manifestations in cognition, event-related potentials and aging. Acta Psychologica (Amst) 101(2-3): 129-58.

Louis-Dam A, Kandel S, Orliaguet J-P (2000) Anticipation motrice et anticipation perceptive. Psychologie Française 45: 333-342.

Loula F, Prasad S, Harber K, Shiffrar M (2005) Recognizing people from their movement. Journal of Experimental Psychology Human Perception and Performance 31(1): 210-20.

Martel L, Bidet-Ildei C, Coello Y (2011) Anticipating the terminal position of an observed action: Effect of kinematic, structural, and identity information. Visual Cognition 19(6): 785-98.

Montagne B, Kessels RP, Frigerio E, De Haan EH, Perrett DI (2005) Sex differences in the perception of affective facial expressions: do men really lack emotional sensitivity?. Cognitive Processing 6(2): 136-41.

Nishitani N, Hari R (2000) Temporal dynamics of cortical representation for action. Proceedings of the National Academy of Sciences USA 97(2): 913-8.

Pavlova M, Guerreschi M, Lutzenberger W, Sokolov AN, Krageloh-Mann I (2010a) Cortical response to social interaction is affected by gender. Neuroimage 50(3): 1327-32.

Pavlova M, Sokolov A (2000) Orientation specificity in biological motion perception. Perception and Psychophysics 62(5): 889-99.

Pavlova M, Wecker M, Krombholz K, Sokolov AA (2010b) Perception of intentions and actions: gender stereotype susceptibility. Brain Research 1311: 81-5.

Rizzolatti G, Fabbri-Destro M (2008) The mirror system and its role in social cognition. Current Opinion in Neurobiology 18(2): 179-84.

Rizzolatti G, Fadiga L, Gallese V, Fogassi L (1996a) Premotor cortex and the recognition of motor actions. Brain Research Cognitive Brain Research 3(2): $131-41$.

Rizzolatti G, Fadiga L, Matelli M, Bettinardi V, Paulesu E, Perani D, Fazio F (1996b) Localization of grasp representations in humans by PET: 1. Observation versus execution. Experimental Brain Research 111(2): 246-52.

Schütz-Bosbach S, Prinz W (2007). Perceptual resonance: action-induced modulation of perception. Trends in Cognitive Sciences 11(8): 349-55.

Shipley, TF (2003) The effect of object and event orientation on perception of biological motion. Psychological Science 14(4): 377-80.

Sirigu A, Cohen L, Duhamel JR, Pillon B, Dubois B, Agid Y (1995) A selective impairment of hand posture for object utilization in apraxia. Cortex 31(1): 41-55.

Stoet G (2010) Sex differences in the processing of flankers. Quarterly Journal of Experimental Psychology (Colchester) 63(4): 633-8. 
Thompson J, Parasuraman R (2011) Attention, biological motion, and action recognition. Neuroimage 59(1): 4-13.

Thornton IM, Vuong QC (2004) Incidental processing of biological motion. Current Biology 14(12): 1084-9. 
